\title{
An Organic FET with an Aluminum Oxide Extended Gate for $\mathrm{pH}$ Sensing
}

\author{
Tsukuru Minamiki, ${ }^{1,2}$ Tomohito Sekine, ${ }^{3}$ Michio Aiko, ${ }^{1}$ \\ Shiwei $\mathrm{Su}^{1}{ }^{1}$ and Tsuyoshi Minami ${ }^{*}$ \\ ${ }^{1}$ Institute of Industrial Science, The University of Tokyo, \\ 4-6-1 Komaba, Meguro-ku, Tokyo 153-8505, Japan \\ ${ }^{2}$ Biomedical Research Institute, National Institute of Advanced Industrial Science and Technology (AIST), \\ 1-1-1 Higashi, Tsukuba, Ibaraki 305-8566, Japan \\ ${ }^{3}$ Research Center for Organic Electronics (ROEL), Yamagata University, \\ 4-3-16 Jonan, Yonezawa, Yamagata 992-8510, Japan
}

(Received August 13, 2018; accepted September 28 2018)

Keywords: organic FETs, aluminum oxide, thin films, reactive ion etching, $\mathrm{pH}$ sensors

Herein, we report on the development of an organic FET (OFET)-based pH sensor. To accomplish the electrical sensing of $\mathrm{pH}$, the designed OFET has an extended-gate electrode consisting of a surface-oxidized aluminum film. As a result, the fabricated device linearly responds to $\mathrm{pH}$ change in the basic region $(\mathrm{pH}>9)$. Importantly, the organic device has superior low-cost processability, portability, and compact integration, indicating that the OFET with the metal-oxide-based electrode could be utilized as on-site monitoring systems for environmental risk assessment.

\section{Introduction}

The development of easy-to-use and inexpensive chemical sensors has become increasingly important because chemical information in the fields of medical treatment and environmental conservation should be collected and analyzed readily on-site. For example, environmental $\mathrm{pH}$ values change with the influx of polluted wastewater, ${ }^{(1)}$ which massively affects the biological activity of bacteria, plankton, plant, and fish. Hence, the development of $\mathrm{pH}$ monitoring tools is required for environmental risk assessment. One of the most conventional methods of $\mathrm{pH}$ detection is the use of $\mathrm{pH}$ test strips. These are widely used for $\mathrm{pH}$ detection because of their portability and low cost. ${ }^{(2,3)}$ However, with such paper-based assays, the quantitative determination of $\mathrm{pH}$ values is difficult to achieve. Additionally, the colorimetric response to $\mathrm{pH}$ changes in the strips is irreversible, meaning that these are not suitable for the continuous measurement of $\mathrm{pH}$. In that regard, ion-sensitive electrodes (ISEs) or ISFETs are developed for $\mathrm{pH}$ sensing. ${ }^{(4)}$ These devices can be easily integrated into compact sensing devices. Moreover, the quantitative and continuous monitoring of $\mathrm{pH}$ can be achieved using these device-based assays. More recently, organic FETs (OFETs) with extended-gate sensing electrodes have also

*Corresponding author: e-mail: tminami@iis.u-tokyo.ac.jp

https://doi.org/10.18494/SAM.2019.2082 
been developed for the detection of environmental pollutants. ${ }^{(5,6)}$ OFETs display excellent low-cost processability and portability, because active materials (i.e., organic semiconductors) in the devices have printability and mechanical flexibility. ${ }^{(7)}$ In addition, OFETs can be readily integrated with radio transmission circuits and indication displays, that is, these are suitable platforms for achieving easy-to-use and inexpensive integrated sensing systems for $\mathrm{pH}$ monitoring. However, the device design and fabrication processes for OFET-based $\mathrm{pH}$ sensors have not been fully established.

In this paper, we report on the development of an OFET with a metal oxide-based electrode capable of sensing $\mathrm{pH}$ changes. To simplify the fabrication process and device structure, an aluminum oxide (AO) film was used for both the sensing membrane and dielectric layer in the fabricated OFET. Herein, we propose the device design and demonstrate the $\mathrm{pH}$ responsiveness of the device.

\section{Materials and Methods}

\subsection{Design and fabrication of OFET}

To achieve the electrical detection of chemical species contained in water, the device should stably operate in aqueous media. However, the electrical characteristics of organic devices are generally unstable under humid conditions, because the thin film of organic semiconductors is easily doped by exposure to electrolyte solutions. ${ }^{(8)}$ Toward that end, we employed the extended-gate structure for the OFET-based sensor device (Fig. 1). ${ }^{(9)}$ In the device structure, the chemical sensing portion in the extended-gate-type OFET is isolated from the drive unit (= a carrier channel region in the OFET), resulting in the prevention of the characteristic degradation for the OFETs. In addition, the sensor device based on the OFET should be used under low applied voltage, because electrolysis causes the instability of the electrical properties of the device. Generally, the operation voltage of the OFET is defined by the capacitance of the gate dielectric. ${ }^{(10)}$ Therefore, we employed an ultrathin dielectric layer that consists of tetradecylphosphonic acid (TDPA, Tokyo Chemical Industry Co., Ltd., Tokyo, Japan) and the AO film. ${ }^{(11,12)}$ The capacitance of the TDPA/AO-based dielectric layer is relatively higher than that of typical paraelectric materials such as insulating polymers, indicating that the designed OFET could operate under a low applied voltage.
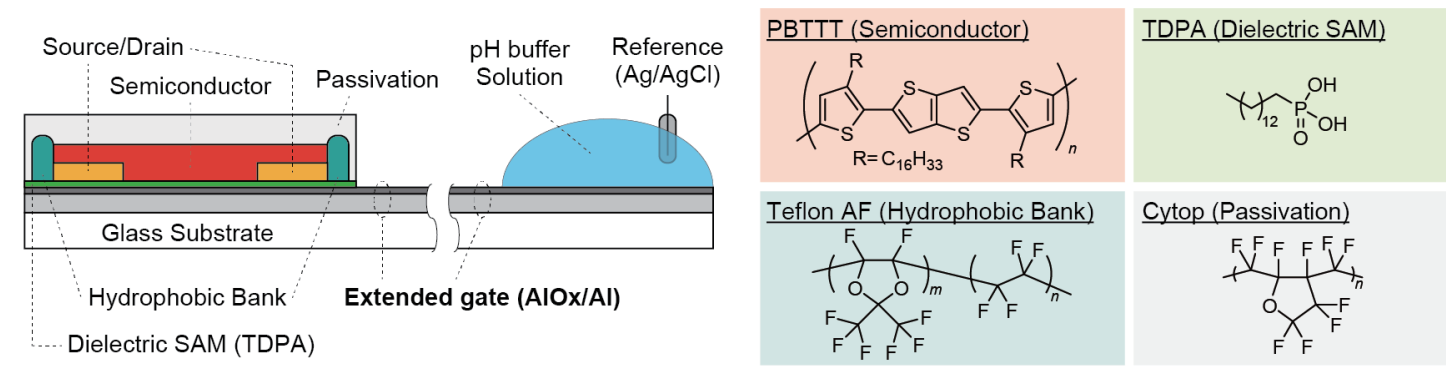

Fig. 1. (Color online) Schematic illustration of OFET with the AO extended gate. 
The fabrication procedure followed that in a previous report. ${ }^{(9)}$ The extended-gate-type OFET was fabricated on a glass substrate (Eagle XG, Corning Inc., New York, USA). Firstly, aluminum (Al, Furuuchi Chemical Co. Ltd., Tokyo, Japan) gate and extended-gate electrodes were vacuum-deposited on the substrate ( $30 \mathrm{~nm}$ thick). The AO layer was formed by oxygen plasma treatment of the Al film surface (plasma power: $300 \mathrm{~W}$ ) using a reactive ion etching (RIE) system (RIE-10NG, SAMCO Inc., Kyoto, Japan). The TDPA monolayer was deposited on the AO surface by immersing the substrate in a 2-propanol solution of TDPA $(1 \mathrm{mM})$. Then, source and drain gold (Au, Tanaka Kikinzoku Kogyo, Tokyo, Japan) electrodes were formed on the gate dielectric layer ( $30 \mathrm{~nm}$ thick). The electrodes were patterned by photolithography and the gap between the source and the drain (= channel length of the OFET) was $20 \mu \mathrm{m}$. To prepare the hydrophobic bank for patterning the semiconductor layer, a Teflon AF (Du Pont, Delaware, USA) was solution-deposited at the channel region of the OFET using a dispenser (IMAGEMASTER 350, Musashi Engineering Inc., Tokyo, Japan). Afterward, a polymeric semiconductor (poly\{2,5-bis(3-hexadecylthiophene-2-yl)thieno[3,2-b]thiophene\}; PBTTT, Merck KGaA, Darmstadt, Germany) ${ }^{(13)}$ in 1,2-dichlorobenzene (FUJIFILM Wako Pure Chemical Co. Ltd., Osaka, Japan) was drop-casted at the channel region. Finally, the OFET was passivated using a spin-coated film of Cytop (CTL-809M, Asahi Glass Co. Ltd., Tokyo. Japan).

\subsection{Electrical measurement of OFET}

The electrical properties of the OFET were measured using a source meter (2602B, Keithley Instruments, $\mathrm{OH}, \mathrm{USA})$. The $\mathrm{pH}$-dependent transfer characteristics of the OFET were measured in a Britton-Robinson buffer solution, which consists of a mixture of $40 \mathrm{mM} \mathrm{H}_{3} \mathrm{BO}_{4}, 40 \mathrm{mM}$ $\mathrm{H}_{3} \mathrm{PO}_{4}$, and $40 \mathrm{mM} \mathrm{CH}{ }_{3} \mathrm{COOH} .{ }^{(14)}$ Gate voltage was applied through a $\mathrm{Ag} / \mathrm{AgCl}$ reference electrode (RE-1S, BAS Inc., Tokyo, Japan), and the $\mathrm{pH}$ of the aqueous solution was titrated by the addition of $\mathrm{HCl}(\mathrm{aq})(0.1 \mathrm{M})$ to the buffer solution. The $\mathrm{pH}$ values of the buffer solution were calibrated using a commercially available $\mathrm{pH}$ meter (D-51, Horiba Ltd., Kyoto, Japan).

\section{Results and Discussion}

\subsection{Low-voltage operation of AO-based OFET}

Initially, we confirmed the low-voltage operation of the fabricated OFET under ambient conditions. Figure 2 shows the basic electrical properties (i.e., transfer and output characteristics) of the device that has the AO dielectric layer (RIE process time: $50 \mathrm{~min}$ ). As a result, the fabricated OFET operated stably and reproducibly under the low applied voltage $(<|3|$ V). These results indicated that the fabricated device could be applied to the chemical sensor device.

\subsection{Surface characterization of extended gate}

To inspect the formation of the oxidized layer on the Al film on the extended gate, we characterized the surface of the plasma-treated Al film by water contact angle goniometry 


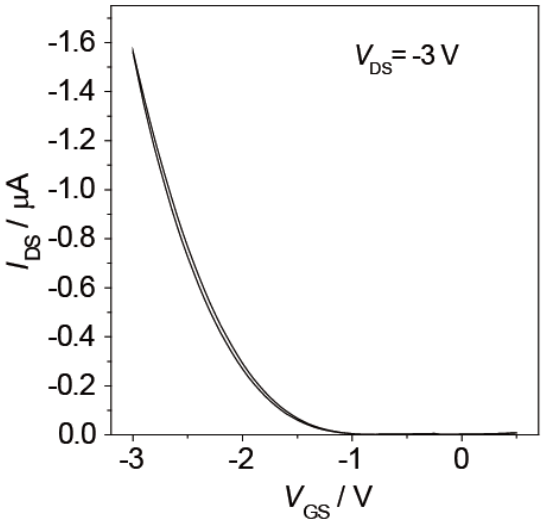

(a)

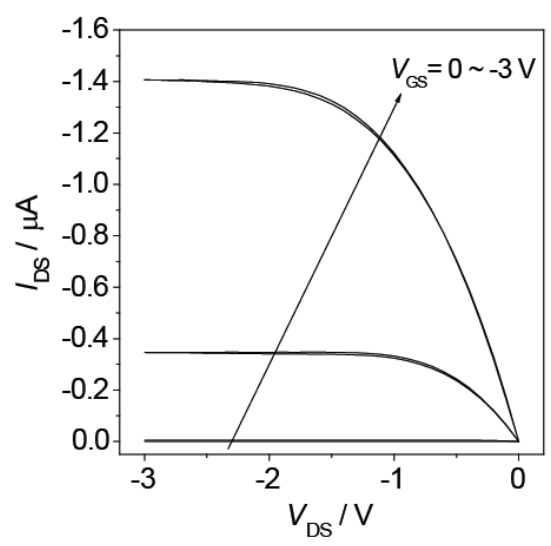

(b)

Fig. 2. Electrical characteristics of low-voltage operable OFET. (a) Transfer characteristics of fabricated OFET. (b) Output characteristics of the same OFET.

(CAG) and X-ray photoelectron spectroscopy (XPS). The CAG measurement was performed to estimate the status of the progress of the surface oxidation of the Al film associated with increasing duration of the RIE process [Fig. 3(a)]. Water droplets were captured using a FACE CA-X goniometer (Kyowa Interface Science Co., Ltd., Saitama, Japan) and the volume of a droplet was about $2 \mu \mathrm{L} .{ }^{(15)}$ The water contact angle at the Al surface was markedly decreased with the lapse of process time [Fig. 3(b)], suggesting that the Al surface was covered with a hydrophilic oxide film (i.e., AO film). Importantly, the observed tendency of wettability changes before and after the RIE process almost coincided with the previous report about the oxygen-plasma-treated Al foil. ${ }^{(16)}$

The XPS measurements were carried out using an ULVAC Phi Model 5600 spectrometer (ULVAC-PHI Inc., Kanagawa, Japan) with AlK $\alpha$ radiation. Figure 4 shows the XPS spectra at A12p regions of the treated Al films under different process times. Two distinct peaks were obtained in each of the spectra. It is worth noting that the main peak $(\sim 75.5 \mathrm{eV})$ is a feature of aluminum oxide and/or hydroxide, and the shoulder peak $(\sim 72.5 \mathrm{eV})$ is attributed to the presence of the Al metal. ${ }^{(16)}$ The relative intensities between the metallic and oxidic Al2p peaks varied with increasing the RIE process time, supporting the fact that the oxygen composite at the Al gate surface (i.e., AO) was generated by the RIE treatment. Taken together, the CAG and XPS results indicate that the $\mathrm{AO}$ layer was successfully formed on the Al extended-gate surface.

\section{3 pH responsiveness of OFET with $\mathrm{AO}$ extended gate}

We have investigated the $\mathrm{pH}$ responsiveness in terms of the electrical characteristics of the OFET with the AO extended gate. The conductance in the OFET channel (=semiconductor/gate dielectric interface) should be affected by changes in the states of the surface potential of the extended gate, ${ }^{(17)}$ meaning that the deprotonation/protonation of the hydroxy moieties at the AO film surface could affect the electrical characteristics of the fabricated device. The electrical 


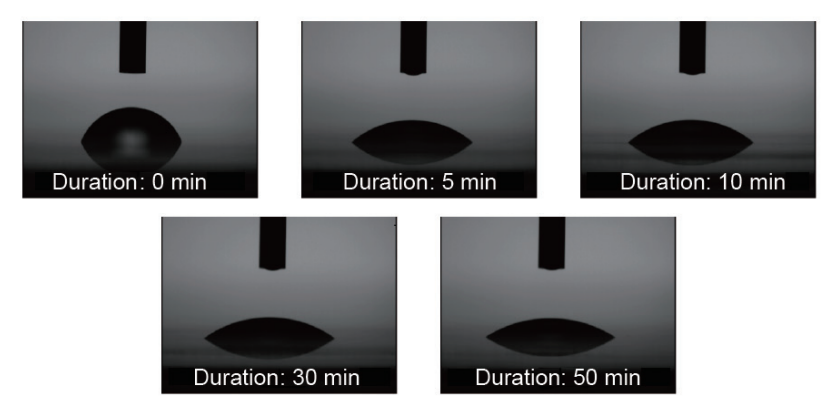

(a)

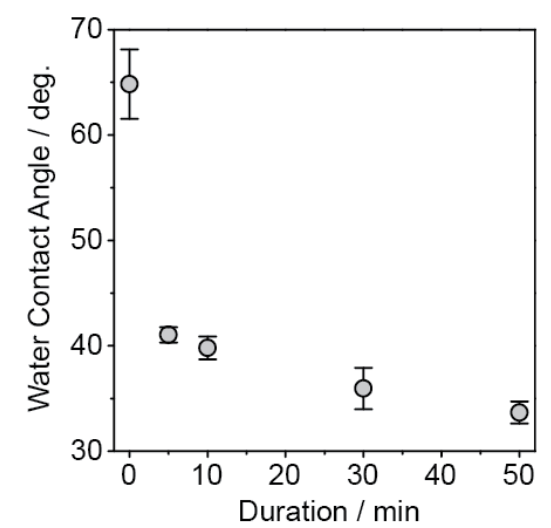

(b)

Fig. 3. (a) CAG measurements of Al film. (b) Effect of RIE duration on water contact angle of Al surface.

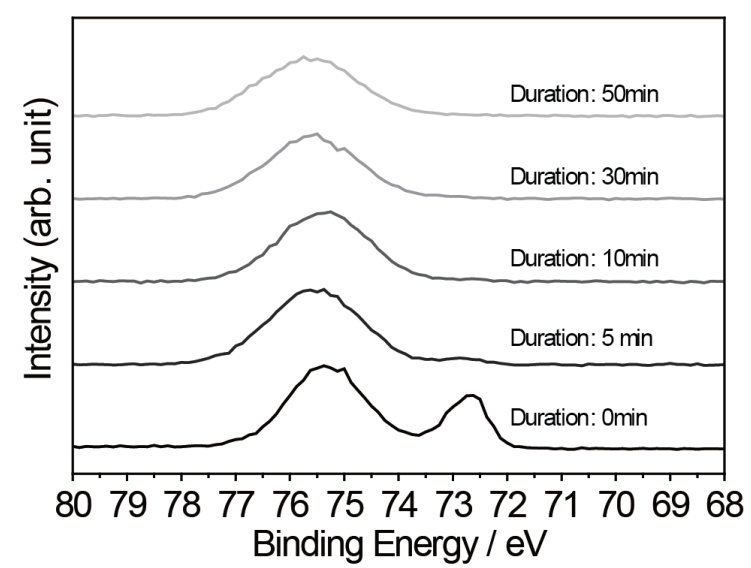

Fig. 4. XPS spectra of A12p regions of the Al extended gate. An electron energy analyzer was operated with a pass energy of $29.35 \mathrm{eV}$.

parameter of the OFET-based sensor was extracted from changes in threshold voltage $\left(V_{T H}\right)$. The $V_{T H}$ value of the OFET at the saturation region is estimated using the following equation: ${ }^{(10)}$

$$
I_{D S}=(W / 2 L) \mu C\left(V_{G S}-V_{T H}\right)^{2},
$$

where $I_{D S}$ is the drain current, $W$ is the channel width $(500 \mu \mathrm{m}), L$ is the channel length $(20 \mu \mathrm{m})$, $\mu$ is the field-effect mobility, and $V_{G S}$ is the gate applied voltage. To achieve the $\mathrm{pH}$ monitoring system based on the OFET, the electrical stability of the device characteristics is crucially important. The extended-gate-type OFET passivated with the Cytop thin film showed high stability under atmospheric conditions for at least 14 days (Fig. 5), indicating that the fabricated device can be utilized for $\mathrm{pH}$ monitoring. 


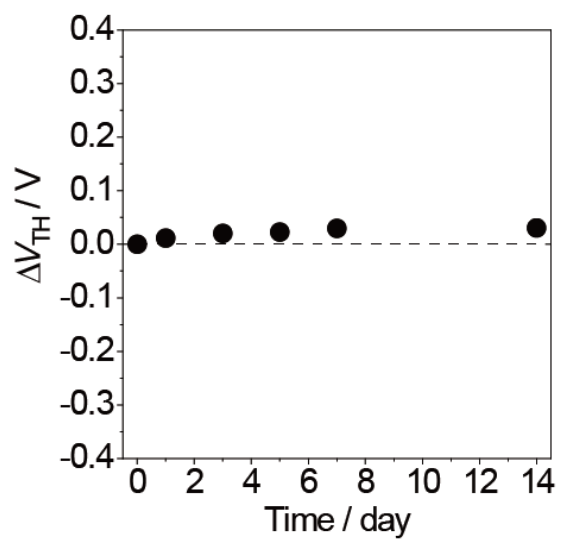

Fig. 5. Time-dependent changes in $V_{T H}$ in the fabricated OFET. The device was stored under ambient conditions.

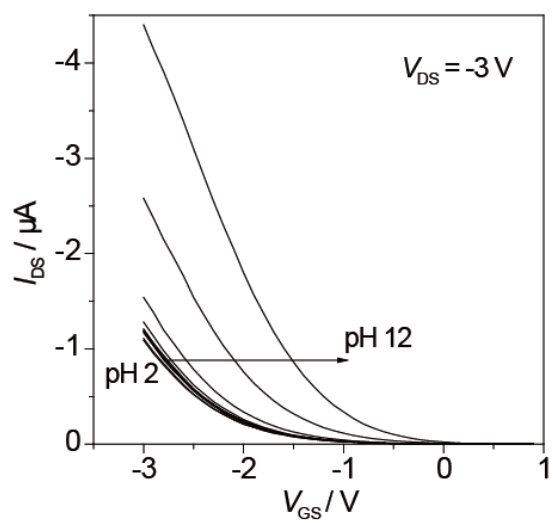

(a)

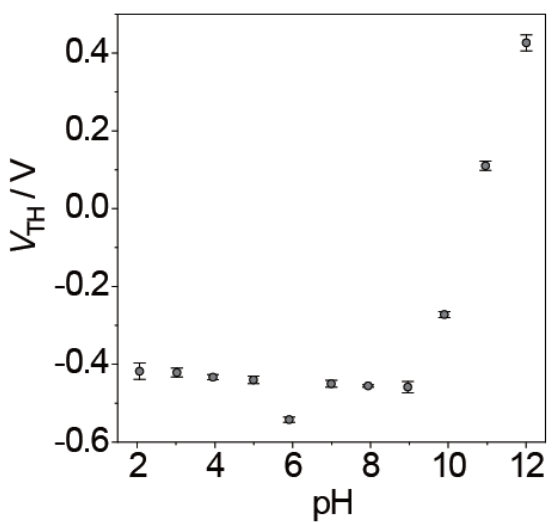

(b)

Fig. 6. (a) Transfer characteristics $\left(I_{D S}-V_{G S}\right.$ curves) of the extended-gate-type OFET upon a change in $\mathrm{pH}$ in a Britton-Robinson buffer solution at $25{ }^{\circ} \mathrm{C}$. The $I_{D S}-V_{G S}$ curves were collected within 1 min after the addition of $\mathrm{HCl}(\mathrm{aq})(0.1 \mathrm{M})$ to the buffer solution. (b) $\mathrm{pH}$ dependence of the threshold voltage of the OFET with a BrittonRobinson buffer solution at $25^{\circ} \mathrm{C}$. Three measurements were carried out for each point.

Finally, we carried out the $\mathrm{pH}$ titration using the OFET device. The transfer curve of the OFET dramatically shifted with changes in $\mathrm{pH}$ at a basic region $(\mathrm{pH}>9)$ [Fig. 6(a)]. Figure 6(b) shows the relationship between the extracted $V_{T H}$ of the OFET and the $\mathrm{pH}$ values of the buffer solution. We obtained a linear relationship in the basic region $(\mathrm{pH}>9)$. The observed $\mathrm{pH}$ responsiveness of the OFET with the AO extended gate was similar to that of anodized AO films, which were reported previously. ${ }^{(18)}$ In addition, Vittoz et al. reported that the hydrophilicity of the AO surface is enhanced in the basic $\mathrm{pH}$ region $(\mathrm{pH}>9),{ }^{(19)}$ supporting the idea that the transfer curve shift of the OFET is related to the deprotonation of the hydroxy moieties at the AO film surface. The linear response range of the OFET to $\mathrm{pH}$ changes covered the basic region ( $\mathrm{pH} 9-12$ ), indicating that the fabricated device could be utilized to monitor the pollution of industrial or household effluent water. ${ }^{(20)}$ Overall, we concluded that the surfaceactivated $\mathrm{Al}$ gate is a suitable component for $\mathrm{pH}$ sensing utilizing the OFET. 


\section{Conclusions}

In conclusion, we successfully demonstrated electrical $\mathrm{pH}$ sensing utilizing the AO-functionalized OFET. The observed $\mathrm{pH}$ responsiveness was derived from the protonation/deprotonation of the hydroxy moieties of the oxidized Al film surface. While we have utilized an $\mathrm{AO}$ film as a representative example of sensing membranes for $\mathrm{pH}$ in this research, we could also functionalize other metal-oxide films on the extended gate in the OFET. For example, the $\mathrm{TiO}_{2}$ film can be utilized not only as a pH-responsive membrane ${ }^{(21)}$ but also as a gate dielectric for the low-voltage operation of OFETs. ${ }^{(22)}$ Importantly, OFETs can be fabricated by low-cost processes on flexible thin films. ${ }^{(23)}$ Thus, we believe that the OFET has a potential application in an on-site sensing system for $\mathrm{pH}$ monitoring. Further development of easy-to-use and inexpensive sensors based on OFETs is being carried out in our laboratory.

\section{Acknowledgments}

This work was financially supported by the Japan Society for the Promotion of Science (JSPS KAKENHI, Grant No. 17H04882). We also acknowledge Asahi Glass Co., Ltd., for supplying the Cytop fluoropolymer.

\section{References}

1 G. E. Hofmann, J. E. Smith, K. S. Johnson, U. Send, L. A. Levin, F. Micheli, A. Paytan, N. N. Price, B. Peterson, Y. Takeshita, P. G. Matson, E. D. Crook, K. J. Kroeker, M. C. Gambi, E. B. Rivest, C. A. Frieder, P. C. Yu, and T. R. Martz: PLoS One 6 (2011) e28983. https://doi.org/10.1371/journal.pone.0028983

2 J. Hu, S. Q. Wang, L. Wang, F. Li, B. Pingguan-Murphy, T. J. Lu, and F. Xu: Biosens. Bioelectron. 54 (2014) 585. https://doi.org/10.1016/j.bios.2013.10.075

3 S. Ahmed, M.-P. N. Bui, and A. Abbas: Biosens. Bioelectron. 77 (2016) 249. https://doi.org/10.1016/ j.bios.2015.09.038

4 N. Moser, T. S. Lande, C. Toumazou, and P. Georgiou: IEEE Sens. J. 16 (2016) 6496. https://doi.org/10.1109/ JSEN.2016.2585920

5 T. Minami, T. Minamiki, and S. Tokito: Jpn. J. Appl. Phys. 55 (2016) 04EL02. https://doi.org/10.7567/ JJAP.55.04EL02

6 Y. Sasaki, T. Minami, T. Minamiki, and S. Tokito: Electrochemistry 85 (2017) 775. https://doi.org/10.5796/ electrochemistry.85.775

7 H. Sirringhaus: Adv. Mater. 26 (2014) 1319. https://doi.org/10.1002/adma.201304346

8 D. Kumaki, T. Umeda, and S. Tokito: Appl. Phys. Lett. 92 (2008) 093309. https://doi.org/10.1063/1.2890853

9 T. Minamiki, T. Minami, R. Kurita, O. Niwa, S. Wakida, K. Fukuda, D. Kumaki, and S. Tokito: Appl. Phys. Lett. 104 (2014) 243703. https://doi.org/10.1063/1.4883739

10 Z. Bao and J. Locklin: Organic Field-Effect Transistors (CRC Press, Boca Raton, 2007).

11 H. Klauk, U. Zschieschang, J. Pflaum, and M. Halik: Nature 445 (2007) 745. https://doi.org/10.1038/ nature 05533

12 K. Fukuda, T. Hamamoto, T. Yokota, T. Sekitani, U. Zschieschang, H. Klauk, and T. Someya: Appl. Phys. Lett. 95 (2009) 203301. https://doi.org/10.1063/1.3259816

13 I. McCulloch, M. Heeney, C. Bailey, K. Genevicius, I. MacDonald, M. Shkunov, D. Sparrowe, S. Tierney, R. Wagner, W. Zhang, M. L. Chabinyc, R. J. Kline, M. D. McGehee, and M. F. Toney: Nat. Mater. 5 (2006) 328. https://doi.org/10.1038/nmat1612

14 H. T. S. Britton and R. A. Robinson: J. Chem. Soc. 0 (1931) 1456. https://doi.org/10.1039/JR9310001456

15 S. Tatara, Y. Kuzumoto, and M. Kitamura: Jpn. J. Appl. Phys. 55 (2016) 03DD02. https://doi.org/10.7567/ JJAP.55.03DD02

16 B. R. Strohmeier: J. Vac. Sci. Technol., A 7 (1989) 3238. https://doi.org/10.1116/1.576342 
17 P. Bergveld: Sens. Actuators, B 88 (2003) 1. https://doi.org/10.1016/S0925-4005(02)00301-5

18 J.-L. Lin, Y.-M. Chu, S.-H. Hsaio, Y.-L. Chin, and T.-P. Sun: Jpn. J. Appl. Phys. 45 (2006) 7999. https://doi. org/10.1143/JJAP.45.7999

19 C. Vittoz, M. Mantel, and J. C. Joud: J. Adhes. 67 (1998) 347. https://doi.org/10.1080/00218469808011116

20 A. G. Heath: Water Pollution and Fish Physiology (CRC Press, Boca Raton, 1995).

21 M. Simić, L. Manjakkal, K. Zaraska, G. M. Stojanović, and R. Dahiya: IEEE Sens. J. 17 (2017) 248. https://doi. org/10.1109/JSEN.2016.2628765

22 Y. Lu, W. H. Lee, H. S. Lee, Y. Jang, and K. Cho: Appl. Phys. Lett. 94 (2009) 113303. https://doi. org/10.1063/1.3097010

23 K. Fukuda and T. Someya: Adv. Mater. 29 (2017) 1602736. https://doi.org/10.1002/adma.201602736 